\title{
Stage IIIC Hilar Cholangiocarcinoma AJCC v8
}

National Cancer Institute

\section{Source}

National Cancer Institute. Stage IIIC Hilar Cholangiocarcinoma A/CC v8. NCI Thesaurus. Code C134750.

Stage IIIC includes: Any T, N1, M0. N1: One to three positive lymph nodes typically involving the hilar, cystic duct, common bile duct, hepatic artery, posterior pancreatoduodenal, and portal vein lymph nodes. M0: No distant metastasis. (AJCC 8th ed.) 